\title{
VISUAL ENVIRONMENT OF COGNITIVE GRAPHICS FOR END-TO-END ENGINEERING PROJECT-BASED EDUCATION
}

\author{
Andrey I. Vlasov ${ }^{1 *}$, Ludmila V. Juravleva ${ }^{1}$, Vadim A. Shakhnov ${ }^{1}$ \\ ${ }^{1}$ Bauman Moscow State Technical University, Russia
}

This article is concerned with the prospects for the implementation of synchronous technology for knowledge processing and transfer by visual methods in the context of end-to-end engineering project-based training. A formal characterization of information about complex systems based on the visual cognitive meta-language VI-XML, forming a synchronous project-based educational environment, is proposed. This environment is a set of methods, using visualization metaphors, while the object under study is presented from different points of view and levels of detail (expertise). Visualization metaphors form the mechanisms for processing and transferringknowledge by comparing abstract or real objects to visually perceptible images. Visual modeling languages, in turn, are formed by fixed sets of metaphors and rules for construction of visual models from them. The proposed approach to the presentation of models underlies the universal visual modeling environment, which provides a single interface for creating and editing visual conceptual, structural-functional, and object models. It provides the ability to encapsulate the levels of visual modeling in a single closed hierarchy, accompanying the stages of system analysis and design. The experience of using a universal visual modeling environment in the frame of practical work and group design has proven itself to be good for the solution of educational problems in the field of knowledge transfer in a visual form that is easily interpreted by students.

Key words: Visual modeling, Project-based education, Formalized knowledge processing, Synchronous technologies, VI-XML meta-language.

\section{INTRODUCTION}

The issues of engineering training in terms of the exponential growth of the volume of information and increasing uncertainty require the use of effective methods of knowledge transfer. The solution of systems engineering, design and technological problems should be considered interconnected in the form of a single and inseparable logistic chain of the product life cycle. Modern production systems are characterized by deep integration of the whole complex of works on design, production, planning, marketing, implementation, operation and utilization of products in the context of synchronous technologies and a single information and control environment. This was the prerequisite for the formation of a new scientific direction - "design and technological informatics", and allowed moving to the knowledge model of formalization of production activities [1].

The modern nature of the consideration of complex production systems in the concept of Industry 4.0 defines the problem of analyzing the ongoing production processes, the labor intensity of building information systems for the formal description, storage, and processing of knowledge about their objects and processes [2]. The technological business environment has pressed entrepreneurs to adopt information systems to overcome operating deficiencies [3]. SMEs can play a vital role in assisting and fostering entrepreneurial activity with a special focus on information technology businesses [4].

A variety of effective approaches to knowledge transfer is implemented through a variety of visual tools and models [2-9]. Comprehensive training in engineering specialties is largely based on visual tools that ensure the creation, transmission, and application of mechanisms for the formalization and manipulation of knowledge in a single semantic interdisciplinary and interspecific hierarchy, providing a single and complete description of the product life cycle processes $[10,11]$. The postulate "one picture replaces 1000 words, and one diagram 1000 drawings"has already become classic. Among the levels of visual models of knowledge representation about production systems, first of all, it is required to single out conceptually-abstract [6], structural-functional [7], and object models [8]. The research on the methods of synthesis of a set of valid, reproducible and systemic methods and means of visual description of the processes occurring in complex systems is relevant. New visual modeling approaches will be able to include in the subject of their activities almost all levels of organization of matter: from the molecular nature of matter $(\mathrm{N}-$ nano) to the nature of life $(B-$ bio), the nature of mind $(C-\operatorname{cogno})$ and information exchange processes ( $I-$ info) i.e. to be a means for efficient description of NBIC systems.

First, at each level, there are objects of the same nature, but the presented descriptions of the model of different levels are not synchronized (the problem of synchronization). One of the main objectives of the development of integrated visual modeling systems is to synchronize the parametric and structural composition of interconnected objects at various levels. Secondly, another 
aspect is the problem of cognition, which is caused by the heterogeneity of the methods used in modern visual models for obtaining and storing knowledge about the subject area. Thirdly, the problem of convergence, which is caused by the complexity of the interpenetration of technologies (conceptual, structural-functional, logical and physical levels of the model), when the boundaries between the individual levels are effaced, and many interesting solutions are located at the intersection of the levels of visual models. New visual modeling tools can be safely applied to NBIC systems, which will include the tools for representation of almost all levels of organization of matter. Fourthly, the problem of encapsulation at this stage of development is due to the fragmentation of visual analysis and its isolation from use in designing at different stages of the life cycle. This determines the low efficiency of the use of visual methods, in particular in the analysis of technological processes, while they provide a description of the individual modules of the social-production system and do not have the means to migrate these models at different levels of expertise.

The search for a solution to these problems is currently focused on the use of visual modeling and design as the main tool for generating, storing and processing knowledge on a specific subject area. The creation of rules for the description of concepts and judgments will make it possible to create a language for the description of engineering and technological knowledge [12]. The authors propose such a language as a subset of XML and call it $\mathrm{I}-\mathrm{XML}$ (Intelligence XML), orienting it to the visual representation of NBIC systems.

\section{LITERATURE REVIEW}

Obviously, having found the ways to solve the above problems on the basis of visual design methods and system analysis, an adequate methodology for a formalized integrated presentation and management of technical, industrial, economic, social and psychological knowledge in complex systems can be offered. Herein, visual modeling is understood as a set of methods that use visualization metaphors, suggest representing an object from different points of view and can be used to develop and evolve the object of modeling [1, 2]. The metaphors of visualization are the comparison of abstract or real objects of visually perceptible images. The languages of visual modeling, in turn, are formed by fixed sets of metaphors and the rules for constructing visual models from them. The term "visual design" means the use of visual expressions (graphs, drawings, icons, tables), which are elements of a graphic language, in the design process. That is, this term refers to those systems that allow representing knowledge about the object of design in two(or more) dimensional form.

The concepts of one subject area often correlate with the concepts of another one, or vice versa, the concepts with the same designata have different characteristics in different contexts. Ensuring a clear relationship and unity of interpretation of concepts is one of the most important tasks solved at the initial conceptual stages of modeling. The formation of concepts occurs as a result of abstraction, which reveals therelations of independence, differentiation,and integration between concepts [13-15]. The concepts are independent if their attributes do not overlap. If the two concepts have common attributes, then there is a differentiation of concepts. If all the attributes of one concept are the attributes of another concept, then they are integrated. The following abstractions are known [8, 16-18]: generalization (specialization), typification (specification), aggregation (decomposition), and association (individuality). Generalization and typification, as well as the inverse specialization and concretization of them, express the commonality of the concepts that appear during differentiation. Aggregation and association, and their reverse decomposition and individualization reveal the integration of the concepts. In generalization, a new concept is generated on the basis of one or several similar concepts, when the generated concept retains the common attributes of the original concepts, but ignores their insignificant differences. Generalization is the generation of a concept based on the intersection of the schemes of generalized concepts and the extended union of their extensions.

In decomposition, the concept - aggregate is divided into the aggregated concepts included in it. Aggregation is a limiting case of association, in which all possible links are present (only a part of links is established during association): several associations can be defined on the same set of concepts, while their aggregation is unique [13 18].

A conceptual structure is a set of concepts for which the formation (abstraction) methods are given. The carrier of the conceptual structure is the set of concepts, and its signature is the set of mappings of generalization, typification, aggregation, and association [13-18]. Unlike the semantic network and conceptual-ontological approach, where different types of relationships are defined on concepts that carry different semantic load, the conceptual structure is defined by a set of concepts with four types of mappings, the only purpose of which is to show the way a concept is formed. The conceptual structure is close to the extended ERmodel ("entity-relationship" model), but in the ERmodel the elements are not concepts, but data types. To ensure a continuous modeling process, from conceptual to informational modeling, it is necessary to implement a mechanism to transfer the conceptual structure components into the data model components, while the conceptual structure itself must meet the requirements of completeness and consistency. A conceptual structure will be called incomplete if there are concepts used in the conceptual structure, but not explicitly defined in it. The conceptual structure is contradictory if there is an entity described as belonging to and not belonging to the same concept. In order to reduce the time required for elimination of contradictions in the next design stages, the synthesized conceptual structure must be verified. The development of verification meth- 
ods for conceptual structures is one of the important objectives of building complex tools for visual modeling. At the same time, the conceptual analysis results can be easily formalized and subjected to elementary checks for completeness and consistency.

Visual modeling tools can be effectively used both in auditing existing production systems and in solving educational problems in the training of modern engineers. Firstly, such tools can provide information on the design of electronic means (EM). This causes the complexity of the compact description of the objects under study as a whole and requires the use of hierarchical decomposition. Secondly, visual tools should be able to describe process models.

\section{METHODS}

Visualization of the complex systems design is understood as the phenomenon of the formal description of the system design process and the finished project in the form of a universal calligrapher with the possibility of decomposition and simulation of variants. For this purpose, a set of techniques (notations) of use of the graphical models (graphical notation) and techniques of formal textual description (textual notation), together with the means of interactive human-machine interaction, is created. This provides a visual and detailed presentation of the characteristics of objects in the design process, expert analysis and online decision-making [12].

The visual design methods are based on visual languages and methods (rules - notations) of the model development based on them. A visual language is defined as a language that systematically uses visual meanings to describe its main objects in textual and graphic notations. The conceptual, structural-functional, logical and physical levels of modeling are singled out (Fig. 1). Analyzing the interrelation of visual models shown in Figure 1 , it should be noted that the lower level represents the specific abstractions; it contains directly the physical elements of the production process, decomposed to the atomic level - the level of technological transition and the elements of information systems corresponding to them. At higher levels, the decisions are made within the competence of groups, sites, workshops, etc. up to the level of top management. At the same time, the objective of formal description tools for processes and elements of each level is to provide the necessary information for this level without excessive detailing. At the upper levels, conceptual-abstract methods prevail, at the middle ones, the structural-functional and object-based methods prevail (when presenting the concrete abstractions).

The conceptual-ontological or structural-functional approaches are often used to study business processes, while the object analysis is used to describe information flows and systems. As for the creation of a universal vi-

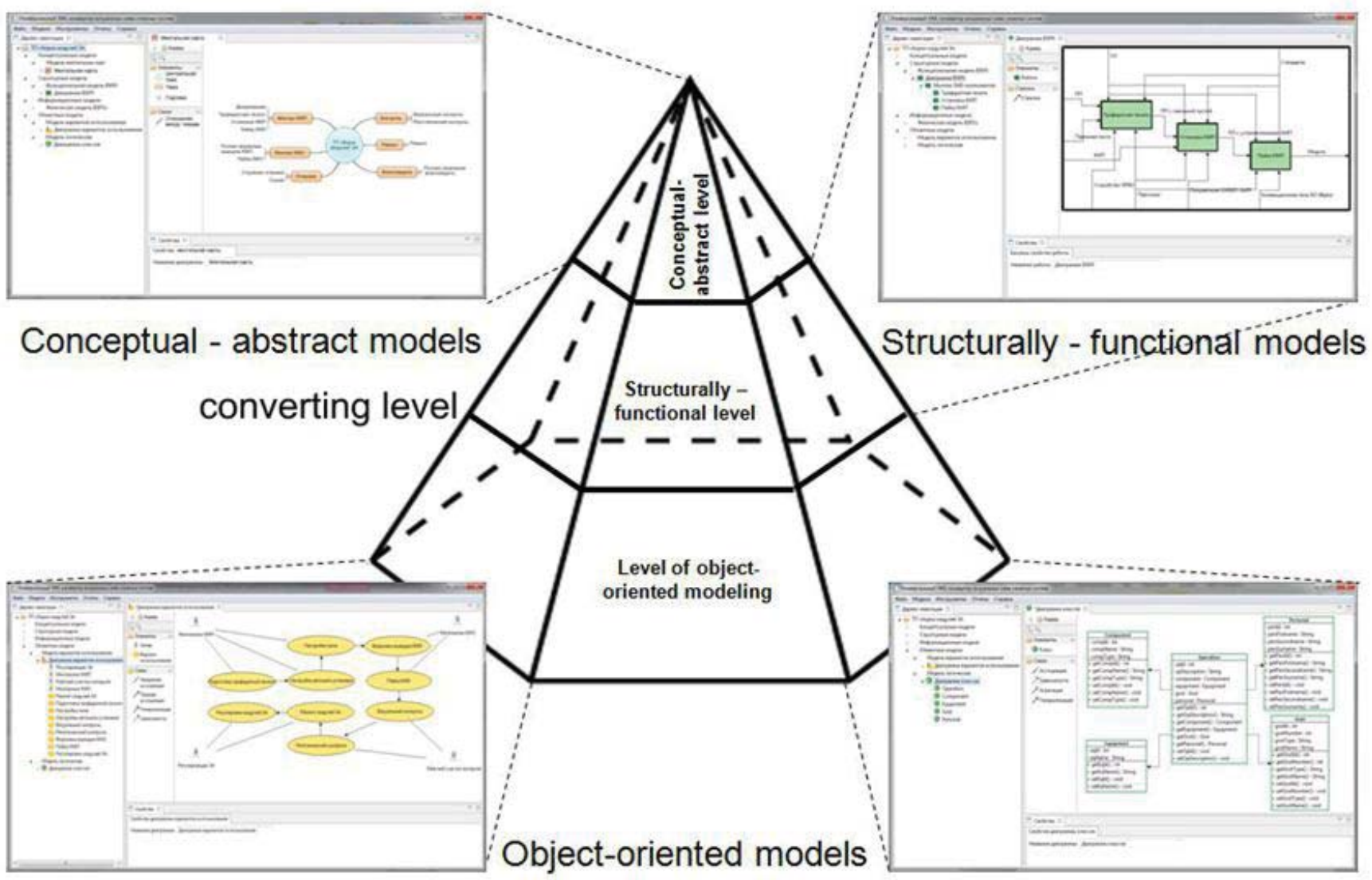

Figure 1: The relationship of the levels of visual modeling [7] 
sual design methodology that takes into account all aspects, it is required to assess the degree of interrelation of conceptual and object analysis.

Conceptual analysis is some generalization of the objective and therefore the statement about the possibility of synthesizing the mechanism of transforming the conceptual model into the objective one with the preservation of completeness and consistency is true. Graphically, the relationship between the components of the conceptual and object models can be represented in the form of a hexagonal close-packed lattice, which determines the principles of the functioning of the mechanisms for transforming models. As a result, it can be argued that the use of the conceptual model (and its particular implementations, structural-functional, object, and the like models) for a complete description of the subject area is sufficient [12-19]. As the core of the system, the authors use a conceptual model, supplemented by a description of the solution of one or several applied tasks:

Program: = Conceptual model (Structure + Syntax + Semantics) + Solution to the problem.

The conceptual structure and syntax of the concepts are described herein in a metalanguage, declared in the context technology, and the description of the semantics and problems to be solved is performed in a specialized subject language defined in the conceptual model.

\section{RESULTS}

The language of formalized description of synchronous visual models $\mathrm{VI}-\mathrm{XML}$ is based on a unified graph structure. The vertices of the graph of the VI model are represented herein as a concept describing the objects and their parameters, and the edges of the graph are represented herein as the judgments describing the relationship of objects. Then the visual model can be described in XML, the elements of which are the concepts and judgments given in Figure 2. In general, the VI-XML file has the following structure [7]. The root element of knowledge contains one element of ideas and opinions, and they, respectively, contain any number of elements of idea or opinion. After analyzing the structure of VI-XML, the following can be concluded: this language is effective for knowledge description, but does not fully meet the objectives of its visual presentation. A simulated system can be defined as a collection of objects and connections between them. Widely used notations (IDEF0 and $\mathrm{UML}$, etc.) graphically represent the diagrams, where the components are represented by different blocks (for example, activity IDEF0), and the links are usually represented by different types of lines (arrows or interface arcs IDEF0) or determined by nested objects. From the point of view of $\mathrm{VI-XML}$, the objects in diagrams are the concepts, and the links are the bundles of judgments.

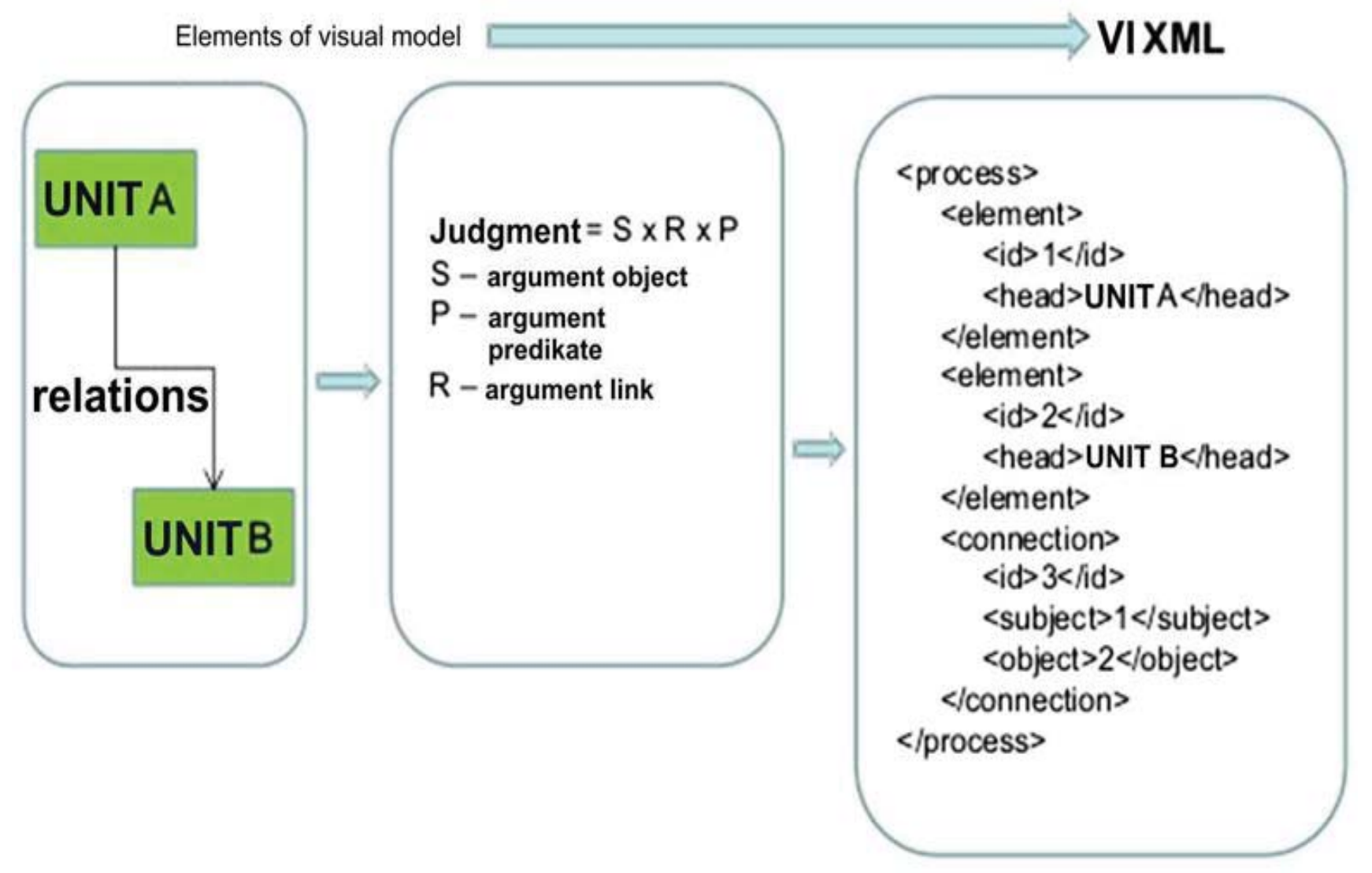

Figure 2: Scheme of formalization of information by means of VI-XML 
The difference between the notations in this case is that each of them breaks the system up into concepts and judgments according to different criteria.

The implementation of visual models is dominated by: conceptually abstract, structural-functional, object and component approaches. In order to consider all aspects of modeling at each of the levels of expertise, it is necessary to take into account the degree of interconnection between conceptual and object analysis. Graphically, such a relationship can be represented in the form of a hexagonal conceptual model in the form of a hexagonal close-packed lattice (Figure 3) [12]. It illustrates the principles of encapsulating models of different levels of expertise (detail). Figure 3 shows one segment of such a lattice; in general, it is synthesized for each space of the conceptual domain, forming a cellular conceptual structure. This construction of the model description language forms the basis of a universal visual modeling environment that provides a single interface for creating and editing visual models in common and new graphical languages. The proposed approach, based on the hexagonal conceptual model [12], determines the ability to combine all levels of visual modeling into a single closed hierarchy that accompanies both process analysis and the construction of information and control modules (Figure 3).

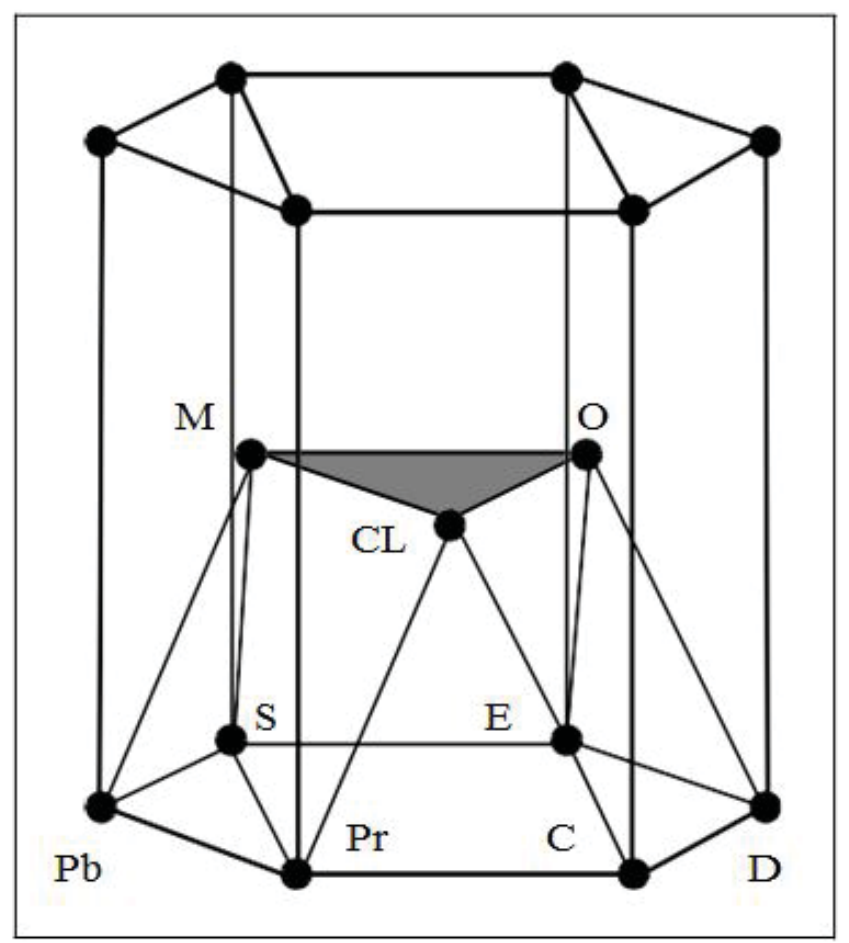

Figure 3: Hexagonal model of encapsulation of conceptual and object analysis of the subject area in the form of a hexagonal close-packed lattice (one lattice segment is shown):

O - Object; $M$ - Method, CL - class, Pb-Problematics, $S$ - Semantics, Pr-Pragmatics, C - Concept, D - Designatum, E-Entity), i, $k$ - levels of areas.
For a graphic representation of the problem area, a flat hexagonal structure formed by the Frege triangle ("Essence - Designatum - Concept") and the triangle "Problems - Pragmatics - Semantics" (Figure 3) [12-18] are introduced. Horizontal links define the links between the components of a model of a separate subject subspace, and vertical links form generalizations of the components of the entire subject area as a whole. In the Frege triangle, an entity is understood as a stable and unique representation of the subject area, perceived by a certain set of attributes [16-18]. Such attribute, as a designated entity, is characterized by its many meanings and has some problematic interpretation (semantic role).

The concept is represented by a set of entities (i.e. there is a generalization connection between them), united on the basis of common attributes. The concepts are given by the scheme, intensional and extensional. The designatum, or a symbolic representation of a concept, is a linguistic unit that carries some meaning in semantic terms. It may denote some specific entity syntactically.

The concept scheme is set by a set of attributes, in which the concept is defined [13-15]. The attributes are interpreted as concepts on which schemes are defined. The intensional, or the content of the concept, is represented by a set of values of interrelated attributes, making it possible to distinguish the entities belonging to a concept from other entities of the subject domain. Extensionality, or the scope of a concept, is considered as a set of entities belonging to the concept.

Usually, the concepts are used not in an absolute, but in a relative sense, called pragmatics [16-18]. The pragmatics of the concept is revealed with the knowledge of the subject area. The problematics specify the semantics of the concept to its pragmatics. As a result, a meaningful interpretation of the concept in a certain subject area can be presented in the form of a hexagonal conceptual and ontological representation (see Figure 3). Thus, it can be argued that for each subject area there is a hexagonal concept interpretation concept.

When analyzing complex systems, in particular, complex social-production systems, one has to deal with various components (technological, social, informational, etc.), each of which forms its own subject area. Consequently, a generalized conceptual interpretation of the concepts in the analysis of complex social and production systems can be represented as a multi-level packed hexagonal structure, each of the planes of which characterizes a specific subject area (see Figure 3).

Using such a model, it is possible to link the concepts with different levels of expertise in XML-like meta-language. As noted above, in terms of I-XML, the objects in the diagrams are the concepts, and the links are the bundles of judgments. The difference between the notations in this case is that each of them breaks the system into the concepts and judgments according to different criteria (which are taken into account as knowledge components). This construction of the model description 
language forms the basis of a universal visual modeling environment that provides a single interface for creating and editing visual models in common and new visual languages. The proposed approach determines the possibility of combining all levels of visual modeling into a single closed hierarchy that accompanies both structural decomposition, process analysis, and the construction of information and control modules. This makes it possible to implement a universal visual design environment.

\section{DISCUSSION}

Information and Communications Technologies (ICTs) include modern tools of knowledge-sharing and communication [19-20]. A certain lexical stock of technical terms and their definitions are used by the specialists for the solution of professional problems. The volume of vocabulary, its content and figurative conformity are determined by the specialty and the relevant subject area, as well as the level of education and job responsibilities of the specialists. Regardless of this, there is a lexical minimum of terms that provides professional understanding at all levels of professional activity. This allows solving professional problems competently and efficiently with minimal expenditure of working time and eliminates the redundancy of discussions, additions, and clarifications.

In the framework of training technical specialists for the development of programs of study in applied disciplines and training materials, it is important to know the volume of technical terms that students have in mind when they begin to study these disciplines. One of the forms for determining the conformity of the figurative representation of terms and their definitions is the compilation of thematic visual glossaries by the students. The students use an unlimited variety of images for the terms, from which they choose the one that best corresponds to their idea of the object defined by this term.

The approbation of the considered approaches to the visual formalization of knowledge was carried out at N.E. Bauman Moscow State Technical University in the framework of the course "Instrument Engineering Technology"(undergraduate). Second-year undergraduate students were offered a list of fifty technical terms and their definitions in the discipline "Instrument Engineering Technology" for studying and visualization, which were grouped in chronological order as they appeared in the educational material in 14 groups (Figure 4). The random sample shown in the figure is a representative part of the population. In this case, the law of distribution of an attribute in the sample corresponds to the law of distribution of this attribute in the general population. In assessing the representativeness of the sample, the information about the population can be qualitative and quantitative. A qualitative assessment of the representativeness of the sample to identify the figurative understanding of technical terms and their definitions by second-year students was based on the results of several pilot studies, during which the similarities and divergences in the figurative representations of technical terms were studied by the second-year students of different cohorts. The

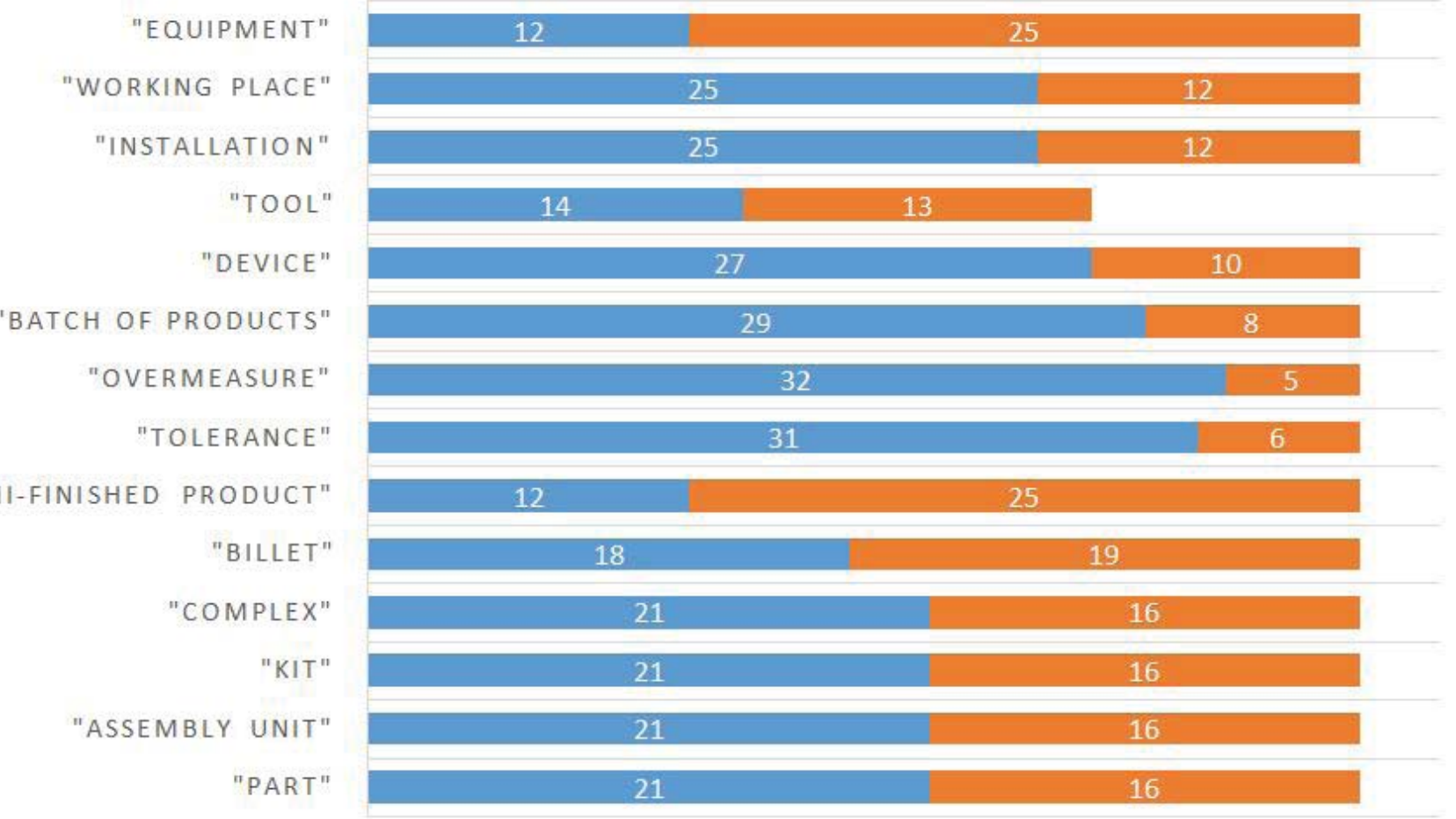

Figure 4: The results of the study of the figurative (visual) understanding of technical terms by the second-year students (the number of visualized terms corresponding to the subject area (blue color), the number of visualized terms not corresponding to the subject area (brown color)). 
similarity of the representations of students from different groups of different cohorts testifies to the system in preparing students for their future profession as part of vocational guidance and makes it possible to judge the volume of their lexical stock of technical terms. According to the experience, the study of such pilot groups, numbering 20-30 people, allows predicting accurately the results obtained in surveys of 1-3 thousand people. In the study of the field of figurative understanding of technical terms, a survey of 80 second-year students of one cohort was conducted. The presented sample of 37 respondents for this study is representative. According to the analysis of Figure 4, the group of terms that form an area of figurative understanding at the stage of the study of the discipline under consideration includes only the terms: "overmeasure", "tolerance" and "batch of products"; their images are highly informative. Ignorance of the rest of terms (the complexity of correct interpretation of the image) creates difficulties in mastering the discipline and mutual understanding of the teacher and students, and therefore requires contextual adaptive learning. The thematic visualized glossaries were used both in the assessment of the initial level of preparation (before giving lectures on the subject) and in the assessment of the final competencies (final, upon completion of training and modularly). This approach has proven itself to be good in assessing the trainees' professional lexical competencies. The proposed technique has also proven itself good in the solution of educational problems in the courses on systems engineering, robotics [21] and other multi-integrated disciplines [22-24] during knowledge transfer in the visual form easily interpretable by students (including foreign students). The particular efficiency of the use of cognitive graphics tools should be noted when teaching foreign students. This approach significantly reduces the time taken by students to overcome the language barrier, contributes to the formation of a professional vocabulary and improves the quality of learning.

\section{CONCLUSION}

As a result of the work, the concept of implementing a visual educational environment in the implementation of project-based learning methods, providing a single interface for the creation and editing of visual domain models at different levels of expertise with the tools of basic graphical languages is proposed. The language of the formalized description of visual models $\mathrm{VI-XML}$ is proposed. The presented solutions provide the synchronism of the technology of subject area description and allow overcoming the fragmentation and isolation of the application of visual analysis at different levels of production systems modeling. The considered approach was actively used in the implementation of educational technologies based on project-based teaching methods. The use of a universal visual design environment has proven itself to be good in the educational process in the training of design engineers and technologists.

\section{ACKNOWLEDGEMENT}

The research was conducted with the support of the Ministry of Science and Education of Russia within the framework of the project under the Agreement No.2.4176.2017/PCh.

\section{REFERENCES}

1. Vlasov, A.I., Ganev, Yu.M., Karpunin, A.A. (2015). System analysis of "lean production" by visual modeling tools. Information Technologies in Design and Production, no. 4 (160),19-24.

2. Koznov, D.V. (2004). Languages of visual modeling: software design and visualization.Publishing House of St. Petersburg State University, St. Petersburg.

3. Akhter, F. (2017). Unlocking digital entrepreneurship through technical business process. Entrepreneurship and Sustainability Issues, vol. 5, no. 1, 36-42. https://doi.org/10.9770/jesi.2017.5.1(3)

4. Abbas, S.A. (2018). Entrepreneurship and information technology businesses in economic crisis. Entrepreneurship and Sustainability Issues, vol. 5, no. 3, 682-692. https://doi.org/10.9770/jesi.2018.5.3(20)

5. Balybin, V.M., Muromtsev, D.Yu., Muromtsev, Yu.L., Orlova, L.P. (2004). Information technologies for designing radio-electronic means.TSTU, Tambov.

6. Koznov, D.V. (2012). Teaching to write software engineering documents with focus on document design by means of MIND MAPS. Proceedings of the IASTED International Conference on Computers and Advanced Technology in Education, CATE 2012, p. 112-118.https://doi.org/10.2316/P.2012.774-036

7. Demin, A.A., Vlasov, A.I. (2017). Visual methods of formalization of knowledge in the conditions of the synchronous technologies of system engineering. ACM International Conference Proceeding Series, 2017, no. 3166098. https://doi. org/10.1145/3166094.3166098

8. Booch, G., Rumbaugh, J., Jacobson, I. (2004). The unified modeling language reference manual, 2nd ed. Addison-Wesley.

9. Koznov, D.V., Larchik, E.V., Terekhov, A.N. (2015). View to view transformations in domain specific modeling. Programming and Computer Software, vol. 41, no. 4, 208-214.https://doi.org/10.1134/ S0361768815040039

10. Shakhnov, V.A., Vlasov, A.I., Rezchikova, E.V., Zinchenko, L.V. (2013). Visual learning environment in electronic engineering education. Proc. International Conference on Interactive Collaborative Learning (ICL),p. 389-398. https://doi.org/10.1109/ICL.2013.6644605 
11. Shakhnov, V.A., Juravleva, L.V., Vlasov, A.I. (2019). Concept of automated support to problem: modular vocational training. Handbook of Research on Engineering Education in a Global Context. IGI Global, p. 101-114.

12. Vlasov, A.I. (2016). The concept of visual analysis of complex systems in the context of synchronous design technologies. Sensors and Systems, no. 8-9 (206), 19-25.

13. Vyhovanec, V.S. (2009a). Applied conceptual analysis. Proc. International Scientific-Practical Conference "Large Systems Management". IPU RAN, Moscow, p. 62-65.

14. Vyhovanec, V.S. (2009b). Methods of analysis of large-scale production. Conceptual analysis and modeling.Proc. International Scientific-Practical Conference "Management of Large-Scale Systems Development". IPU RAN, Moscow, p. 308-317.

15. Vyhovanec, V.S. (2011).On the concept of the concept.Proc. IX International Scientific-Practical Conference "Large Systems Management-2011". IPU RAN, Moscow, p. 39-42.

16. Pospelov, D.A. (1975). Large systems. Situational management. Znaniye, Moscow, p. 64.

17. Pospelov, D.A. (1986). Situational management: theory and practice. Nauka, Moscow, p. 288.

18. Lomako, E.I. (2008). System encyclopedia. Moscow.
19. Peregudov, F.I., Tarasenko, F.L. (1989). Introduction to system analysis. VSH, Moscow.

20. Allabouche, K., Diouri, O., Gaga, A., El Amrani El Idrissi, N. (2016). Mobile phones' social impacts on sustainable human development: case studies, Morocco and Italy. Entrepreneurship and Sustainability Issues, no. 4(1): 64-73. https://doi.org/10.9770/ jesi.2016.4.1(6)

21. Yudin, A.V., Vlasov, A.I., Salmina, M., Sukhotskiy, V. (2019). Challenging intensive project-based education: short-term class on mobile robotics with mechatronic elements. Advances in Intelligent Systems and Computing,vol. 829, 79-84.https://doi. org/10.1007/978-3-319-97085-1_8

22. Velidzhanashvili, A.Z. (2003). Some issues of creative activity and the role of information technologies in their formation. Education Sciences and Psychology,no. 2(3), 44-48.

23. Koutamanis, A., Heuer, J., Könings, K.D. (2017). A visual information tool for user participation during the lifecycle of school building design: BIM. European Journal of Education,vol. 52,no. 3, 295-305. https://doi.org/10.1111/ejed.12226

24. Solomons, S.N. (1989). Conceptual models in industrial design. Ph.D. Thesis. Council for National Academic Awards, United Kingdom. 